\title{
CYP3A4 ubiquitination by gp78 (the tumor autocrine motility factor receptor, AMFR) and CHIP E3 ligases *
}

\author{
Michael K. Pabarcus ${ }^{a}$, Nicholas Hoe ${ }^{a}$, Sheila Sadeghi $^{a}$, Cam Patterson $^{b}$, Emmanuel \\ Wiertz $^{\mathrm{C}, \mathrm{d}}$, and Maria Almira Correia ${ }^{\mathrm{a}}{ }^{*}$
}

${ }^{a}$ Department of Cellular and Molecular Pharmacology, University of California San Francisco, 600 16th Street, N572F/Box 2280, Genentech Hall, San Francisco, CA 94158-2280, United States ${ }^{b}$ Carolina Cardiovascular Biology Center, University of North Carolina, Chapel Hill, NC 27599, United States 'Department of Medical Microbiology, Leiden University Medical Center, 2300 RC Leiden, The Netherlands dDepartment of Medical Microbiology, University Medical Centre Utrecht, Heidelberglaan 100, 3584 CX Utrecht, The Netherlands

\begin{abstract}
Human liver CYP3A4 is an endoplasmic reticulum (ER)-anchored hemoprotein responsible for the metabolism of $>50 \%$ of clinically prescribed drugs. After heterologous expression in Saccharomyces cerevisiae, it is degraded via the ubiquitin (Ub)-dependent $26 \mathrm{~S}$ proteasomal pathway that utilizes Ubc7p/Cue1p, but none of the canonical Ub-ligases (E3s) Hrd1p/Hrd3p, Doa10p, and Rsp5p involved in ER-associated degradation (ERAD). To identify an Ub-ligase capable of ubiquitinating CYP3A4, we examined various in vitro reconstituted mammalian E3 systems, using purified and functionally characterized recombinant components. Of these, the cytosolic domain of the ER-protein gp78, also known as the tumor autocrine motility factor receptor (AMFR), an UBC7-dependent polytopic RING-finger E3, effectively ubiquitinated CYP3A4 in vitro, as did the UbcH5a-dependent cytosolic E3 CHIP. CYP3A4 immunoprecipitation coupled with anti-Ub immunoblotting analyses confirmed its ubiquitination in these reconstituted systems. Thus, both UBC7/gp78 and UbcH5a/ CHIP may be involved in CYP3A4 ERAD, although their relative physiological contribution remains to be established.
\end{abstract}

\section{Keywords}

CYP3A4; Cytochrome P450 ubiquitination; ERAD; E3 ubiquitin ligases; E3s; UBC7; gp78; CHIP; HRD1; TEB4

\footnotetext{
Cytochrome P450 3A4 (CYP3A4) ${ }^{1}$ is the dominant human liver P450 enzyme responsible for the metabolism of over $50 \%$ of clinically relevant drugs [1,2]. In common with the other members of the hepatic endoplasmic-reticulum (ER)-anchored P450 family, CYP3A4 is a monotopic hemoprotein with its $\mathrm{N}$-terminus embedded in the ER-membrane with the bulk of its structure exposed to the cytosol. Like its rat liver orthologs, CYP3A4 is inducible not only via transcriptional activation but also via substrate-mediated stabilization of its degradation $[3,4]$, thereby revealing that hepatic $\mathrm{CYP}^{2} \mathrm{~A}^{2}$ levels may be regulated through modulation of its proteolytic degradation.
}

\footnotetext{
${ }^{\star 2}$ Supported by NIH grants GM44037 (MAC) and DK26506 (MAC).

(C) 2009 Published by Elsevier Inc.

*Corresponding author. Fax: +1 415476 5292. almira.correia@ucsf.edu (M.A. Correia). .
} 
CYP3A degradation both in vivo and in vitro largely occurs via the ubiquitin (Ub)-dependent $26 \mathrm{~S}$ proteasomal pathway (UPD) [5]. Our studies in intact rats, isolated hepatocytes in suspension and/or primary culture have shown that both native and structurally damaged/ suicidally inactivated CYPs $3 \mathrm{~A}^{2}$ are UPD substrates, subscribing to a typical ER-associated degradation (ERAD) process, wherein the protein is ubiquitinated, dislocated from the ER and delivered to the cytosolic $26 \mathrm{~S}$ proteasome for proteolytic destruction, apparently chaperoned by the AAA ATPase p97-Ufd1-Npl4 complex [6-9]. This process is considerably enhanced in hepatocytes in suspension or culture after treatment with the CYP3A mechanism-based inactivator DDEP (3,5-dicarbethoxy-2,6-dimethyl-4-ethyl-1,4-dihydropyridine) [6,7,9]. P450 UPD could also be documented in an in vitro reconstituted system that included CYP3A4 on an ER-membrane scaffold, and Ub-, ATP/ $\mathrm{MgCl}_{2}$-supplemented rat liver cytosolic Fraction II (FII) as the source of the ubiquitination and 26S proteasomal machinery [8]. Furthermore, in an effort to identify the molecular participants involved in this ERAD process, we have examined the degradation of heterologously expressed CYP3A4 in wild type Saccharomyces cerevisiae and mutants containing defined genetic lesions in various yeast ERAD components [10-12]. We found that CYP3A4 ERAD in yeast requires the ERAD-associated soluble E2 Ub-conjugating enzyme Ubc7p and its membrane-anchor Cue1p, the mammalian p97 homolog Cdc48p-Ufd1p-Npl4 complex, and Rpn1p (Hrd2p), an essential 26S proteasomal cap subunit $[5,13,14]$. However, although CYP3A4 was clearly degraded in an Ubc7p/Cue1p- and Rpn1pdependent ERAD process, none of the canonical yeast ERAD-associated E3 Ub-ligases such as Hrd1p/Hrd3p, Doa10p or Rsp5p, known to participate in the ERAD of integral and lumenal ER and/or cytosolic and nuclear proteins [15-19] could be implicated [5,13,14].

Our identification of Ubc7p/Cue1p as the E2 complex involved in CYP3A4 ERAD [5,13,14], the high evolutionary conservation of mammalian Ubc7p orthologs i.e. murine MmUBC7 (Ube2g2) [20,21], coupled with our previously documented successful ubiquitination of

\footnotetext{
${ }^{1}$ Abbreviations used:

ATP Adenosine triphosphate

CPK creatine phosphokinase

CHIP C-terminal Hsp70-interacting protein

$\mathrm{CuOOH}$ cumene hydroperoxide

CYP3A4 Cytochrome P450 3A4

ER Endoplasmic Reticulum

ERAD ER-associated degradation

DTT dithiothreitol

EDTA ethylenediaminetetraacetic acid

GSH glutathione

gp78/AMFR "glycoprotein" 78/autocrine motility factor receptor

His3A His-tagged CYP3A4

CP phosphocreatine

TAO troleandomycin

Ub Ubiquitin

UPS Ub-dependent 26S proteasomal system

${ }^{2}$ CYPs 3A refer to liver CYPs 3A2, 3A23, 3A18 and 3A9 when the statements are applicable to rats, and human liver CYP3A4, when they are applicable to both species.
} 
cumene-hydroperoxide (CuOOH)-inactivated CYP3A4 in a liver FII-reconstituted system [8] and the recent availability of purified recombinant mammalian E2 and E3 enzymes [22-29], led us to examine several in vitro reconstituted ubiquitination systems to identify a plausible mammalian E3 Ub-ligase involved in CYP3A4 UPD.

We examined three ER-integral UBC7-dependent RING finger E3 Ub-ligases ["glycoprotein" 78/autocrine motility factor receptor (gp78/AMFR), HRD1 and TEB4], and one UBC7independent, UbcH5a-dependent cytosolic E3 (CHIP, C-terminal Hsp70-interacting protein) in in vitro reconstituted CYP3A4 ubiquitination systems. HRD1, one of the human orthologs of yeast Hrd1p Ub-ligase, is involved in the basal degradation of 3-hydroxy-3methylglutarylcoenzyme A reductase, but not in that of its sterol-regulated isoform which utilizes gp78, another yeast Hrd1p ortholog [22-24]. gp78/AMFR is a polytopic protein Nterminally anchored to the ER-membrane with its intrinsic RING-finger Ub-ligase, Cue1-like, UBC7/Ube2g2-binding, substrate recognition and p97-binding regions all situated in its cytosolic C-terminal 309-643 residue (gp78C) domain [27-30]. TEB4 [also known as MARCH-VI], one of the nine human membrane-anchored RING-CH proteins [18,25] and the human ortholog of yeast Doa10p Ub-ligase, is known to autoubiquitylate itself and to be involved in its own proteasomal degradation [25]. RING-finger E3s bind their cognate E2 enzymes, catalyze the preassembly of $\mathrm{Lys}_{48}$-linked polyUb chains and mediate the direct transfer of this nascent polyUb chain from the E2 to a suitable Lys-residue of either a target substrate [31] or itself [22]. Such gp78-catalyzed substrate ubiquitination requires nearly its entire cytosolic C-terminal domain (309-611 residues) [28].

The U-box type E3 CHIP contains an N-terminal tetratricopeptide repeat (TPR) domain that binds Hsc70 and Hsp90 and a C-terminal U-box that functions as a chaperone-dependent Ubligase that links the cytosolic chaperone machinery to UPD [32-35]. CHIP has been shown to ubiquitinate CYP2E1, another hepatic ER-anchored P450 [36]. Our findings described herein reveal that of the four recombinant mammalian Ub-ligase systems examined, gp78 and CHIP both ubiquitinated $\mathrm{CuOOH}$-inactivated CYP3A4 in vitro.

\section{Experimental procedures (materials and methods)}

\section{Chemicals and reagents}

Chemicals and reagents were purchased from the following sources: Adenosine triphosphate (ATP), cumene hydroperoxide ( $\mathrm{CuOOH})$, glutathione (GSH), creatine phosphokinase (CPK), phosphocreatine (CP), were obtained from Sigma-Aldrich, St. Louis, MO; Chaperones Hsp70, Hsc70 and Hsp40 were purchased from Assay Designs (Ann Arbor, MI). Ub-activating enzyme E1 was purchased from BioMol (Plymouth Meeting, PA), Ub-conjugating enzyme E2 UbcH5a was obtained from Boston Biochem (Boston, MA).

\section{CYP3A4}

A cDNA encoding a C-terminally His 6 -tagged CYP3A4 (His3A4) with its N-terminal 3-12 residues deleted, and its Ser18 replaced by Phe, was incorporated into a $\mathrm{pCWori}^{+}$vector, expressed in DH $\alpha$ cells grown in TB media at $37^{\circ} \mathrm{C}$, induced with IPTG, the temperature lowered to $30^{\circ} \mathrm{C}$, and then allowed to grow as described previously [36]. His3A4 was purified using nickel affinity and hydroxylapatite chromatography, as described previously [36]. Stock aliquots were stored at $-80^{\circ} \mathrm{C}$ until use.

\section{Expression plasmids}

Plasmids pGEX-gp78C encoding the human cytosolic C-terminal gp78 domain (residues 309643) [27], and pGEX-MmUBC7 encoding murine UBC7 were kind gifts from Dr. A. M. Weissman (NCI, Frederick, MD). pGEX-4T1-HRD1CT1A and pGEX-4T1-HRD1CT1B, 
respectively encoding 603- and 575-residue domains of the cytosolic human HRD1 C-terminal RING-H2 finger E3 Ub-ligase, were gifts from Dr. Vincent Chau (Pennsylvania State University College of Medicine, Hershey, PA; [22,25]. The plasmids pGEX-TEB4r and pGEXTEB4rC9A encoding the cytosolic RING-finger domains of TEB4 and its inactive mutant TEB4C9A, respectively, were constructed as described [25]. Plasmid pET-CHIPHis ${ }_{6}$ encoding the full length 303-residue human CHIP as a His-tagged fusion inserted in frame into pET-30a vector was constructed as described [32]. The plasmid pGEX-2TK-Ub encoding the GST-Ub fusion protein [37] was donated by Dr. P. Howley (Harvard Medical School, Boston, MA).

\section{E3s and Ub}

The recombinant proteins GST-Ub, GST-UBC7 and GST-gp78C, GST-HRD1CT1A, GSTHRD1CT1B, GST-TEB4ring (r) and GST-TEB4r-C9A were expressed from the above pGEXbased plasmids as fusion proteins with GST incorporated at the N-terminus. Full length Ub and UBC7 were generated after thrombin cleavage of the GST-tag. The cytosolic domains of gp78 and HRD1, abbreviated gp78C and HRD1CT1, respectively, were recovered as active enzymes fused to GST. gp78C contains all the domains required for its E3 Ub-ligase function except its $\mathrm{N}$-terminal ER-membrane-anchor that is dispensable [27,28]. The HRD1CT1B construct differs from its corresponding HRD1CT1A construct in that it is 28 residues shorter in the N-terminal portion of the cytosolic HRD1CT1 domain. For GST-TEB4r and GSTTEB4rC9A, only the RING finger domain of TEB4 was fused to GST, with C9A indicating an inactive mutant. Briefly, plasmids were expressed in Escherichia coli BL21 cells cultured with $2 x$ YT/ampicillin at $37^{\circ} \mathrm{C}$. Cultures were induced at $0.5-0.6 \mathrm{OD}_{600}$ with $1 \mathrm{mM}$ IPTG and harvested after $3-5 \mathrm{~h}$ by centrifugation at $14,000 \mathrm{~g}$ for $20 \mathrm{~min}$. Cell pellets were stored at -20 ${ }^{\circ} \mathrm{C}$. Clarified crude supernatants were prepared by re-suspension of cell pellets in PBS containing $2 \mathrm{mM}$ PMSF, $5 \mathrm{mM}$ DTT, and $1 \mathrm{mM}$ EDTA, treatment with lysozyme, sonication and centrifugation at $10,000 \mathrm{~g}$ for $20 \mathrm{~min}$. Proteins were purified using glutathione (GSH)Sepharose as per the manufacturer's protocol. Further purification of GST-UBC7 was performed with Q-Sepharose Fast Flow using $20 \mathrm{mM}$ Tris-HCl, pH 8.0, with a gradient from 150 to $650 \mathrm{mM} \mathrm{NaCl}$ over 12 column volumes, while GST-HRD1CT1A and GST-HRD1CT1B were purified with Resource Q 6 ml-column using $20 \mathrm{mM}$ Tris-HCl, pH 8.0, with a gradient from 0 to $500 \mathrm{mM} \mathrm{KCl}$. Purified GST-UBC7 was cleaved in vitro with thrombin at $4{ }^{\circ} \mathrm{C}$, overnight. Free GST was removed with GSH-Sepharose, while thrombin was removed using HiTrap Benzamidine columns (AP Biotech). Purified UBC7 was concentrated using a Centricon 4 centrifugal device. His-CHIP was expressed and purified as per a published protocol [32]. Protein concentrations for all samples were determined using BioRad Protein Assay kit.

The expressed recombinant proteins UBC7 (18 kDa), GST-gp78C (63 kDa), His-CHIP (35 kDa), GST-HRD1CT1A (43 kDa observed), GST-HRD1CT1B (40 kDa observed), GSTTEB4r (30 kDa), and GST-TEB4rC9A (30 kDa) were recovered as purified proteins with a few minor contaminant bands as determined by SDS-PAGE analyses. In all cases, the recombinant protein of interest represented the majority component by NIH Image analyses of purified GST-HRD1CT1A, GST-HRD1CT1B, GST-TEB4r, and GST-TEB4rC9A. Purified proteins were stored in aliquots at $-80^{\circ} \mathrm{C}$.

${ }^{32} \mathrm{P}-\mathrm{Ub}$

GST-Ub was radiolabeled using the method described [38]. Briefly, previously purified GSTUb or stored cell pellet, resuspended, lysed, and clarified by centrifugation, was incubated with GSH-Sepharose. The Sepharose was washed, incubated with the protein kinase A (PKA) and $\gamma-{ }^{32} \mathrm{P}-\mathrm{ATP}$ to ${ }^{32} \mathrm{P}$-label Ub, and washed again. Purified ${ }^{32} \mathrm{P}-\mathrm{Ub}$ was obtained after thrombininduced oncolumn cleavage of the bound GST-tag. The supernatant was recovered and thrombin removed as described above. Purified ${ }^{32} \mathrm{P}-\mathrm{Ub}$ was concentrated using a Microcon 4 
centrifugal device and quantified using an extinction coefficient A280 of $1=6.69 \mathrm{mg} / \mathrm{ml}$ [39].

\section{CYP3A4 inactivation}

His3A4 was inactivated for $20 \mathrm{~min}$ at $37^{\circ} \mathrm{C}$ with $0.5 \mathrm{mM} \mathrm{CuOOH}, 1 \mathrm{mM}$ EDTA, $1 \mathrm{mM} \mathrm{GSH}$ in $1 \mathrm{X}$ PBS containing $10 \%$ glycerol. DTT $(5 \mathrm{mM})$ was added at the end to quench the reaction. A $50 \% \mathrm{CuOOH} / \mathrm{EtOH}$ dilution was used to prepare the aqueous solutions. Inactivation was confirmed by the loss of CO-dependent spectral content over the range of 400-500 nm [36; data not shown] relative to a non-CuOOH-treated His $3 \mathrm{~A} 4$ control sample incubated in parallel. Hsc70 $(2.5 \mu \mathrm{M})$, and $\mathrm{Hsp} 40(2.5 \mu \mathrm{M})$ were included during the $\mathrm{CuOOH}$-inactivation of His3A4 used in the CHIP reactions.

\section{Reconstituted CYP3A4 ubiquitination systems}

CYP3A4 ubiquitination was examined exactly as detailed for other ERAD substrates $(22,23$, $31,33,40)$. In those reports, assessment of the relative functional potential of various E3 Ubligases and/or their site-directed mutants in ubiquitinating the ERAD substrate in question, was made in parallel at a time point when the ubiquitination reaction was found to be maximal with optimal concentrations of the components involved. Accordingly, all UBC7-dependent ubiquitination reactions contained the following: E1 $(0.1 \mu \mathrm{M})$, UBC7 $(4 \mu \mathrm{M}),{ }^{32} \mathrm{P}-\mathrm{Ub}(20$ or $167 \mu \mathrm{M}$ as indicated), ATP $(4 \mathrm{mM}), \mathrm{CPK}(20 \mathrm{U} / \mathrm{ml}), \mathrm{CP}(20 \mathrm{mM}), \mathrm{MgCl}_{2}(10 \mathrm{mM})$, DTT (1 $\mathrm{mM})$ and $\mathrm{CuOOH}$-inactivated His3A4 (250 pmol), and one of the following E3s: GST-gp78C $(1 \mu \mathrm{M})$, GST-HRD1CT1A $(10 \mu \mathrm{M})$, GST-HRD1CT1B $(10 \mu \mathrm{M})$, GST-TEB4r $(10 \mu \mathrm{M})$, or its inactive mutant GST-TEB4rC9A $(10 \mu \mathrm{M})$. UbcH5a and His-CHIP-dependent ubiquitination reactions contained: UbcH5a $(1.5 \mu \mathrm{M})$ instead of UBC7, His-CHIP $(4 \mu \mathrm{M})$ as the E3, Hsc70 $(2.5 \mu \mathrm{M})$, and $\mathrm{Hsp} 40(2.5 \mu \mathrm{M})$, in an otherwise identical reaction mixture. In some UbcH5a/ CHIP systems, Hsp70 $(2.5 \mu \mathrm{M})$ was also included instead of CYP3A4 as a substrate. All reactions were incubated at $30^{\circ} \mathrm{C}$ for $90 \mathrm{~min}$, and terminated by the addition of SDS-PAGE sample buffer. They were then heated for $10 \mathrm{~min}$ at $95^{\circ} \mathrm{C}$ and analyzed by SDS-PAGE.

\section{SDS-PAGE and Phosphorlmager analyses}

Ubiquitination reaction mixtures were analyzed by SDS-PAGE on 4-20\% gradient gels at 150 $\mathrm{V}$ for $1 \mathrm{~h}$. Gels were stained with Coomassie brilliant blue R250 for $10 \mathrm{~min}$ and destained with $30 \%$ methanol, $10 \%$ acetic acid, three times, each for $20 \mathrm{~min}$. Gels were dried using BioRad Gel dryer Model 740. Dried gels were exposed to PhosphorImaging screens and visualized using Typhoon scanner and ImageQuant software. Exposures to PhosphorImager screens ranged from $>72 \mathrm{~h}$ for the $\mathrm{Ub}(20 \mu \mathrm{M})$-reactions and $\approx 18 \mathrm{~h}$ for the $\mathrm{Ub}(167 \mu \mathrm{M})$-reactions.

\section{Immunoprecipitation analyses of ubiquitinated CYP3A4}

To establish that CYP3A4 was indeed ubiquitinated, the protein was immunoprecipitated from reactions identical to those detailed above including control incubations in the absence of ATP or CYP3A4. At the end of each reaction, $\mathrm{N}$-ethylmaleimide ( $5 \mathrm{mM}$, final concentration) was added followed by $10 \%$ SDS to a final concentration of $2 \%$ and the samples boiled for $5 \mathrm{~min}$. CYP3A4 was immunoprecipitated with goat anti-CYP3A4 IgG (3 mg) bound to Protein ASepharose CL-4B $(200 \mu \mathrm{L})$ after the mixture was end- to-end rotated at $4{ }^{\circ} \mathrm{C}$ overnight essentially as described $(7,9)$. The immunoprecipitated CYP3A4 was solubilized with $75 \mu \mathrm{L}$ of SDS-PAGE loading buffer containing 5\% SDS, 20\% glycerol, DTT (50 mM) and 5\% $\beta$-ME in $50 \mathrm{mM}$ Tris buffer, $\mathrm{pH} 6.8$, boiled for $5 \mathrm{~min}$, and then equivalent aliquots $(45 \mu \mathrm{L})$ were subjected to SDS-PAGE on 4-20\% Tris-HCl gels. The gels were then dried and exposed to PhosphorImaging screens, and visualized using a Typhoon scanner. 


\section{Immunoblotting analyses of ubiquitinated CYP3A4}

Aliquots of immunoprecipitates from the above reactions were also subjected to SDS-PAGE on 4-20\% polyacrylamide gels and subsequent electrotransfer onto a nitrocellulose membrane as previously described [7,9], with rabbit anti-Ub IgGs as the primary antibody and goat antirabbit alkaline phosphatase-conjugated secondary antibody detection system.

\section{Results}

\section{Functional competence of the recombinant ubiquitinating enzymes}

The functional competence of $\mathrm{UBC} 7$ and the expressed $\mathrm{E} 3 \mathrm{~s}$ in in vitro ubiquitination reactions was first assessed in reconstituted reaction mixtures that excluded $\mathrm{CuOOH}$-inactivated CYP3A4 (Fig. 1). By itself, in the presence of an ATP-generating system, UBC7 was capable of generating Ub-dimers to some extent (Fig. 1). When combined with a cognate E3, the UBC7/ GST-gp78C and UBC7/GST-HRD1CT1A systems were clearly able to catalyze the formation of Ub-dimers and polyUb chains (Ub-oligomers) from E1-activated ${ }^{32} \mathrm{P}-\mathrm{Ub}$ in in vitro systems, resulting in the characteristic high molecular mass (HMM) step-ladder polyubiquitination profile (Fig. 1A and C). On the other hand, the UBC7/GST-HRD1CT1B while enhancing Ubdimer formation appeared relatively less efficient in polyUb chain assembly and/or autoubiquitination, thereby indicating that truncation of $28 \mathrm{~N}$-terminal residues from the cytosolic HRD1CT1A domain impaired these processes (Fig. 1D). Impaired polyUb chain assembly and/or autoubiquitination were also observed with UBC7/GST-TEB4r system (Fig. 1E). As expected, the inactive UBC7/TEB4rC9A mutant used as a control showed no Ub-dimer formation (Fig. 1F). The UbcH5a/His-CHIP system, when similarly examined, indicated a CHIP-dependent polyubiquitination profile, which may reflect CHIP autoubiquitination. This polyubiquitination profile was enhanced on inclusion of the molecular chaperones Hsp70/ Hsc70 (known CHIP targets) and Hsp40 in the reaction mixture (Fig. 1B).

\section{Reconstituted CYP3A4 ubiquitination reactions}

Inclusion of $\mathrm{CuOOH}$-inactivated $\mathrm{CYP} 3 \mathrm{~A}^{3}{ }^{3}$ in the reaction mixture enhanced the HMM protein polyubiquitination profile in the UBC7/gp78C system that was clearly absent when ATP or gp78C was excluded from the system (Fig. 2A). Furthermore, in the presence of CYP3A4, the detection of the ubiquitination signal was extended to a much higher mass range (100-250 $\mathrm{kDa}$ ), consistent with the polyubiquitination of this particular $55 \mathrm{kDa}$ protein. Incubation of $\mathrm{CuOOH}$-inactivated CYP3A4 in an UbcH5a/CHIP reconstituted system supplemented with its required $\mathrm{Hsc} 70$ and Hsp40 chaperones, yielded a HMM ubiquitination profile considerably less pronounced than that seen with the UBC7/gp78C system. The detection of this profile was also dependent on ATP and CHIP (Fig. 2B). By contrast, all the other systems tested failed to show significant CYP3A4 ubiquitination (Fig. 2C-F) and in the case of the UBC7/HRD1CT1A system (and UBC7/HRD1CT1B to a lesser extent), a slight inhibitory effect on the HMM ubiquitination profile was observed upon CYP3A4 inclusion in the reaction mixture (Fig. 2E). Furthermore, increasing the Ub-concentration in the incubations from 20 to $167 \mu \mathrm{M}$, clearly enhanced CYP3A4 ubiquitination by both UBC7/gp78 and UbcH5a/CHIP (Figs. 3A and 4A), but not by HRD1CT1A or TEB4r (data not shown).

\footnotetext{
${ }^{3}$ Unlike in vivo conditions, wherein the difference between native and inactivated CYP3A species is maintained and reflected by their differential degradation rates[6,7,9], purified "native" CYP3A4 and its rat liver CYP3A orthologs do not remain "native" during the entire (>90 $\mathrm{min}$ ) in vitro incubation period at $30^{\circ} \mathrm{C}$ required for $\mathrm{CuOOH}$-inactivation and subsequent ubiquitination reactions. They denature and are thus also susceptible to ubiquitination, albeit more slowly than the $\mathrm{CuOOH}$-inactivated species. Because, non-CuOOH inactivated CYP3A4 does not truly reflect the "native" species, it was not included as an appropriate control in these in vitro incubations.
} 


\section{Confirmation of CYP3A4 ubiquitination by immunoprecipitation/immunoblotting analyses}

To verify that CYP3A4 was specifically ubiquitinated in these reaction mixtures and/or determine the relative extent of this ubiquitination, CYP3A4 was immunoprecipitated with goat anti-CYP3A4 polyclonal IgGs. Reaction mixtures containing ATP but no CYP3A4, or CYP3A4-containing reactions incubated in the absence of ATP were similarly treated to control for non-specific protein interactions. The SDS-PAGE/PhosphorImager analyses of the initial UBC7/gp78-catalyzed reactions in the presence of Ub (167 $\mu \mathrm{M})$ (Fig. 3A) and of CYP3A4 immunoprecipitated from aliquots of these reactions (Fig. 3B) are shown. Immunoprecipitates from complete reactions containing CYP3A4 exhibited a significant HMM ( $>75-250 \mathrm{kDa}$ ) protein polyubiquitination profile, relative to those in its absence or those containing no ATP (Fig. 3B). Immunoblotting analyses of aliquots of these immunoprecipitates with anti-Ub IgGs correspondingly revealed the HMM Ub-CYP3A4ladders, characteristic of ubiquitinated proteins (Fig. 3C). Immunoprecipitation analyses of corresponding reactions carried out with Ub $(20 \mu \mathrm{M})($ Fig. 2A) yielded similar CYP3A4 ubiquitination profiles, albeit considerably less intense than those observed at Ub $(167 \mu \mathrm{M})$ (Fig. 3D).

After comparable immunoprecipitation analyses of UbcH5a/CHIP incubations at Ubconcentrations of $20 \mu \mathrm{M}$ containing CYP3A4 over that of the control sample without CYP3A4 (Fig. 2B), no such clearly enhanced signal at higher molecular weights could be detected irrespective of the length of exposure to PhosphorImager screens (data not shown). However, when a higher Ub-concentration $(167 \mu \mathrm{M})$ was included in the incubations, similar CYP3A4 immunoprecipitation analyses of corresponding $\mathrm{UbcH} 5 \mathrm{a} / \mathrm{CHIP}$ reactions revealed that CYP3A4 was indeed ubiquitinated in the presence but not absence of ATP (Fig. 4). Similar analyses of the UBC7/HRD1CT1A or UBC7/HRD1CT1B systems failed to reveal specific CYP3A4 ubiquitination profile, even at the higher Ub-concentration (167 $\mu \mathrm{M}$; data not shown). The latter was also true of the TEB4r-catalyzed system.

\section{Discussion}

Having established the functional competence of our in vitro reconstituted UBC7-dependent ubiquitination systems, their relative ability to utilize $\mathrm{CuOOH}$-inactivated CYP3A4 as a substrate was assessed. In the absence of CYP3A4, the UBC7/gp78C system was quite robust in Ub-oligomerization, as reflected by the characteristic HMM ubiquitination profile on SDSPAGE/PhosphorImager analyses. This is indicative of nascent polyUb chain assembly that occurs either freely or on an UBC7 molecule as recently proposed [31], and that subsequently may be tagged onto accessible Lys (K)-residues of a GST-E3-RING fusion [22,40] and/or a copurified protein contaminant (Fig. 1). However, inclusion of CYP3A4 in this system not only clearly increased the intensity of the observed ubiquitination signal but also extended its range to higher molecular weights, consistent with the ubiquitination of this $55 \mathrm{kDa}$ CYP3A4 protein (Fig. 2). This profile was further enhanced when the Ub-concentration in the incubation was increased eight-fold to $167 \mu \mathrm{M}$ (Fig. 3A). That CYP3A4 had indeed been ubiquitinated was documented by the considerably enhanced HMM profile on SDS-PAGE analysis of the CYP3A4 species immunoprecipitated from the UBC7/gp78C reaction mixtures by an antiCYP3A4 polyclonal antibody over that from corresponding non-CYP3A4 incubated controls (Fig. 3B). Immunoblotting analyses with anti-Ub IgGs of the CYP3A4 immunoprecipitates revealed the characteristic HMM ladder profile of ubiquitinated proteins, thereby providing further evidence for CYP3A4 ubiquitination (Fig. 3C). These findings are entirely consistent with our previous observation that such HMM profiles reflect ubiquitinated CYP3A4 in in vitro ubiquitination systems with ${ }^{35} \mathrm{~S}$-labeled CYP3A4 [8], or in vivo in hepatocytes treated with mechanism-based CYP3A inactivators $[6,7,9]$. 
Of the other UBC7-dependent E3s tested, the two cytosolic HRD1 C-terminal domain constructs CT1A and CT1B, while fully competent in the elaboration of Ub-dimers and/or polyUb chains, were incapable of significant CYP3A4 ubiquitination, even after the Ubconcentration was raised eightfold. This was also true of the TEB4r E3 and as expected of its RING-finger inactive mutant TEB4rC9A. Thus of the four different UBC7-dependent ERintegral E3 proteins tested, only gp78 was capable of significant CYP3A4 ubiquitination (Figs. $2 \mathrm{~A}$ and $3 \mathrm{~A}$ and D). Interestingly, this human ERAD-associated Ub-ligase is a polytopic ERprotein with six N-terminal transmembrane domains and a cytosolic tail. This tail in addition to the functionally indispensable RING-finger (residues 341-378) also contains an intrinsic Cue-domain homologous to yeast Cue1p (residues 456-497, required for binding Ub rather than UBC7), an UBC7/Ube2g2-docking domain (G2BR, residues 579-600), and a C-terminal p97/VCP-interacting motif (VIM, C-terminal 30 amino acid residues) that is highly conserved in vertebrates $[28,29,41]$. Thus, the identification of gp78 as a dominant CYP3A4ubiquitinating enzyme makes ample sense given the strategic cytosolic localization not only of the bulk of the CYP3A4 molecule, but also of the functional Ub-ligase RING-finger, Ubbinding, UBC7-docking and p97-binding domains of gp78, that would enable efficient CYP3A4 ubiquitination at the cytosolic ER surface before its dislocation by the p97 complex. This gp78 identification is entirely consistent with our previous in vivo findings not only in cultured hepatocytes that identified a role for p97 in CYP3A ERAD [9], but also in $S$. cerevisiae [14] that revealed a dependence of CYP3A4 ERAD on Ubc7p/Cue1p and Cdc48Ufd1/Npl4 complex, the yeast equivalent of the p97-complex. Moreover, it also validates the obligatory requirement for ubiquitination implied by the yeast CYP3A4 ERAD dependence on Ubc7p/Cue1p. Recently, gp78 has been proposed to function as an E4 in CFTR $\triangle$ F508 UPD by extending the polyUb chain length after initial monoubiquitination of that protein by RMA1, another ERAD E3 [42]. We find it noteworthy that in our in vitro reconstituted system, gp78 by itself was capable of polyubiquitinating CYP3A4 and thus played both roles (Fig. 3).

Our findings of the lack of significant CYP3A4 ubiquitination in mammalian in vitro HRD1 and TEB4 systems are also highly consistent with our exclusion of Hrd1p and Doa10p, the yeast E3 orthologs of these mammalian ER-integral E3s, respectively, in CYP3A4 ERAD in S. cerevisiae $[13,14]$. Although arguably, the functional failure of TEB4 and HRD1 to appreciably ubiquitinate CYP3A4 could be attributed to the inability of their RING-finger domains to fully interact with either CYP3A4 or UBC7 after deletion of their membraneanchor, this did not prevent their efficient catalyses of nascent polyUb chains (Fig. $2 \mathrm{C}-\mathrm{E}$, lanes 3 ). Together, these findings once again underscore the close similarity between the yeast and mammalian P450 UPD processes. However, although CYP3A4 ERAD unequivocally occurs in yeast, Hrd1p, the only yeast E3 with at best, limited homology to human gp78 in its membrane spanning and RING-domains [22,27] has been convincingly excluded [13,14]. Thus, the E3 that serves as the human gp78 counterpart in yeast CYP3A4 ERAD remains to be identified.

UbcH5a/CHIP was considerably less efficient than UBC7/gp78C in CYP3A4 ubiquitination at the lower Ub-concentrations $(20 \mu \mathrm{M})$, as judged by the relatively less pronounced HMM profile observed on inclusion of CYP3A4 (Fig. 2B). Corresponding CYP3A4 immunoprecipitation studies revealed no appreciably enhanced CYP3A4 ubiquitination in CYP3A4-supplemented reaction mixtures relative to controls lacking CYP3A4 (Data not shown). However, this very sluggish CYP3A4 ubiquitination was not due to a functionally inactive UbcH5a/CHIP, as replacement of CYP3A4 with Hsp70/Hsc70, documented CHIP target substrates $[43,44]$ in the reaction, resulted in their robust CHIP-mediated ubiquitination (Figs. 1B and 2B). Furthermore, CYP2E1, another hepatic P450, has been previously reported to be ubiquitinated in vitro in an UbcH5a/CHIP-dependent process in the presence of an even lower $(8.3 \mu \mathrm{M}) \mathrm{Ub}$-concentration [35]. This CYP2E1 ubiquitination was greatly enhanced following its $\mathrm{CCl}_{4}$-mediated suicide inactivation [35]. Unlike suicidally inactivated CYP2E1 
which is readily ubiquitinated, $\mathrm{CuOOH}$-inactivated CYP3A4 apparently becomes a functional CHIP target only when the Ub-concentrations are substantially raised >20-fold to $167 \mu \mathrm{M}$ (Fig. 4). Thus, it is unclear whether under physiological conditions CYP3A4 would normally be a CHIP target. Although CYP3A4 is significantly degraded in yeast, no CHIP ortholog apparently exists in this organism thus precluding the direct in vivo assessment of its specific relevance using genetic deletion analyses similar to those used to establish the independence of CYP3A4 ERAD from Hrd1p and Doa10p. However, it is noteworthy, that although yeast CHIP orthologs are unknown, the UbcH5a orthologs Ubc4 and Ubc5 do participate in the degradation of short-lived and abnormal proteins such as the structurally inactivated CYP3A4 $[43,45,46]$.

Together the above findings reveal that of the in vitro mammalian CYP3A4 ubiquitination systems tested, the UBC7/gp78 system is clearly the most efficient, followed by UbcH5a/ CHIP. While these findings unequivocally reveal that CYP3A4 is ubiquitinated, it is unclear whether the electrophoretic migration of this modified CYP3A4 species to molecular weight ranges extending to $>250 \mathrm{kDa}$ reflects its attachment of polyUb chains of variable length $(n=$ 4-20 Ub molecules) onto a single CYP3A4 K-residue, and/or similar ubiquitination of multiple CYP3A4 K-residues. Indeed, of the known P450 sequences

(http://drnelson.utmem.edu/CytochromeP450.html), CYPs 3A are remarkable in their relatively high content of K-residues. Moreover, the CYP3A4 structure reveals that most of its K-residues are indeed on the surface $[47,48]$ and thus eminently accessible to the ubiquitination machinery. While basal ubiquitination of CYP3A proteins is normally detectable [9], this process is greatly enhanced both in vivo and in vitro after their suicide inactivation and structural insults that unravel the $\mathrm{P} 450$ structure and greatly accelerate this process $[6,7,9,49]$. It is conceivable however, that such structural disruption enhances CYP3A4 ubiquitination through exposure of either a concealed P450 molecular determinant "degron" for recognition by these E3 Ub-ligases, or one or more additional and otherwise concealed K-residue(s). Accordingly, the observed differences in CYP3A4 ubiquitination profile by gp78 and CHIP (Figs. 3 and 4) may reflect not only differences in the relative number and/or specific CYP3A4 K-residues targeted by each of these Ub-ligases, but also the length of the specific Ub-chain elaborated on each residue. Identification of the precise CYP3A4 residues targeted by either gp78 or CHIP may provide insight into the mode of their individual CYP3A4 substrate recognition.

In summary, our comparative studies of in vitro reconstituted CYP3A4 ubiquitination systems with the mammalian ER E3s gp78, HRD1 and TEB4 utilizing UBC7/Ube2g2 as their cognate $\mathrm{E} 2$, or the cytosolic CHIP with UbcH5a as its cognate E2 have singled out gp78 as the more dominant CYP3A4 Ub-ligase that is functional at relatively low Ub-concentrations (Figs. 2A and 3). However, CHIP is capable of significant CYP3A4 ubiquitination at higher Ub-

concentrations (Fig. 4). Given the conceivably higher intrahepatic Ub-concentrations than the nominal cellular 10-20 $\mu \mathrm{M}$-concentrations [50], it remains to be determined whether such redundancy of CYP3A4 ubiquitination systems is physiologically relevant and/or cooperative. Alternatively, such an enhanced CHIP-targeting of structurally inactivated CYP3A4 species could be due to molecular chaperone corralling of a particular molecular conformation of the inactivated protein (Fig. 5). Because of the ER-membrane localization of the polytopic gp78 Ub-ligase [27] and the cytosolic localization of CHIP, it is quite conceivable that CYP3A4 ubiquitination occurs sequentially in its ERAD, with gp78 acting initially while CYP3A4 is still anchored N-terminally to the ER-membrane, and CHIP acting after its p97-mediated ERdislocation into the cytosol as shown for rat liver CYPs 3A [9]. Alternatively, differences in the specific conformation of each structurally inactivated CYP3A4 molecule may account for the differential substrate recognition by each of these Ub-ligases. 


\section{Acknowledgments}

The authors wish to sincerely thank Drs. Allan M. Weissman (NCI), Vincent Chau (Pennsylvania State University, Hershey) and Peter Howley (Harvard Medical School) for the generous gifts of expression plasmids, and Dr. Yihong Ye (NIDDK) for valuable advice on gp78 expression and purification. We also thank Dr. Poonam Sansawal for preliminary experiments on protein expression and Dr. YongQiang Wang for his generous assistance with some enzyme purification procedures. We acknowledge the UCSF Liver Center Cores on Molecular Analyses (Spectrophotometry) supported by P30DK26743.

\section{References}

[1]. Guengerich, FP. Cytochrome P450: Structure, Mechanism and Biochemistry. Ortiz de Montellano, P., editor. Kluwer, Academic/Plenum Press; New York: 2005. p. 377-530.

[2]. Correia, MA. Cytochrome P450: Structure, Mechanism and Biochemistry. Ortiz de Montellano, P., editor. Kluwer, Academic/Plenum Press; New York: 2005. p. 619-657.and references therein

[3]. Watkins PB, Wrighton SA, Schuetz EG, Maurel P, Guzelian PS. J. Biol. Chem 1986;261:6264-6271. [PubMed: 3486184]

[4]. Eliasson E, Mkrtchian S, Halpert JR, Ingelman-Sundberg M. J. Biol. Chem 1994;269:18378-18383. [PubMed: 8034584]

[5]. Correia MA, Liao M. Expert Opin. Drug Metab. Toxicol 2007;3:33-49. [PubMed: 17269893]

[6]. Correia MA, Davoll SH, Wrighton SA, Thomas PE. Arch. Biochem. Biophys 1992;297:228-238. [PubMed: 1497342]

[7]. Wang HF, Figueiredo Pereira ME, Correia MA. Arch. Biochem. Biophys 1999;365:45-53. [PubMed: 10222037]

[8]. Korsmeyer KK, Davoll S, Figueiredo-Pereira ME, Correia MA. Arch. Biochem. Biophys 1999;365:31-44. [PubMed: 10222036]

[9]. Faouzi S, Medzihradszky KF, Hefner C, Maher JJ, Correia MA. Biochemistry 2007;46:7793-7803. [PubMed: 17550236]

[10]. Wilhovsky S, Gardner R, Hampton R. Mol. Biol. Cell 2000;11:1697-1708. [PubMed: 10793145]

[11]. Hampton RY. Curr. Opin. Cell Biol 2002;14:476-482. [PubMed: 12383799]

[12]. Kostova Z, Wolf DH. Embo J 2003;22:2309-2317. [PubMed: 12743025]

[13]. Murray BP, Correia MA. Arch. Biochem. Biophys 2001;393:106-116. [PubMed: 11516167]

[14]. Liao M, Faouzi S, Karyakin A, Correia MA. Mol. Pharmacol 2006;69:1897-1904. [PubMed: 16556771]

[15]. Bays NW, Gardner RG, Seelig LP, Joazeiro CA, Hampton RY. Nat. Cell Biol 2001;3:24-29. [PubMed: 11146622]

[16]. Bordallo J, Plemper RK, Finger A, Wolf DH. Mol. Biol. Cell 1998;9:209-222. [PubMed: 9437001]

[17]. Swanson R, Locher M, Hochstrasser M. Genes Dev 2001;15:2660-2674. [PubMed: 11641273]

[18]. Kreft SG, Wang L, Hochstrasser M. J. Biol. Chem 2006;281:4646-4653. [PubMed: 16373356]

[19]. Haynes CM, Caldwell S, Cooper AA. J. Cell Biol 2002;158:91-101. [PubMed: 12105183]

[20]. Tiwari S, Weissman AM. J. Biol. Chem 2001;276:16193-16200. [PubMed: 11278356]

[21]. Fang S, Weissman AM. Cell Mol. Life Sci 2004;61:1546-1561. [PubMed: 15224180]

[22]. Kikkert M, Doolman R, Dai M, Avner R, Hassink G, van Voorden S, Thanedar S, Roitelman J, Chau V, Wiertz E. J. Biol. Chem 2004;279:3525-3534. [PubMed: 14593114]

[23]. Doolman R, Leichner GS, Avner R, Roitelman J. J. Biol. Chem 2004;279:38184-38193. [PubMed: 15247208]

[24]. Song BL, Sever N, DeBose-Boyd RA. Mol. Cell 2005;19:829-840. [PubMed: 16168377]

[25]. Hassink G, Kikkert M, van Voorden S, Lee SJ, Spaapen R, van Laar T, Coleman CS, Bartee E, Fruh K, Chau V, Wiertz E. Biochem. J 2005;388:647-655. [PubMed: 15673284]

[26]. Flierman D, Coleman CS, Pickart CM, Rapoport TA, Chau V. Proc. Natl. Acad. Sci. USA 2006;103:11589-11594. [PubMed: 16868077]

[27]. Fang S, Ferrone M, Yang C, Jensen JP, Tiwari S, Weissman AM. Proc. Natl. Acad. Sci. USA 2001;98:14422-14427. [PubMed: 11724934] 
[28]. Chen B, Mariano J, Tsai YC, Chan AH, Cohen M, Weissman AM. Proc. Natl. Acad. Sci. USA 2006;103:341-346. [PubMed: 16407162]

[29]. Zhong X, Shen Y, Ballar P, Apostolou A, Agami R, Fang S. J. Biol. Chem 2004;279:45676-45684. [PubMed: 15331598]

[30]. Kostova Z, Tsai YC, Weissman AM. Semin. Cell Dev. Biol 2007;18:770-779. [PubMed: 17950636]

[31]. Li W, Tu D, Brunger AT, Ye Y. Nature 2007;446:333-337. [PubMed: 17310145]

[32]. Ballinger CA, Connell P, Wu Y, Hu Z, Thompson LJ, Yin LY, Patterson C. Mol. Cell Biol 1999;19:4535-4545. [PubMed: 10330192]

[33]. Murata S, Minami Y, Minami M, Chiba T, Tanaka K. EMBO Rep 2001;2:1133-1138. [PubMed: 11743028]

[34]. McDonough H, Patterson C. Cell Stress Chaperones 2003;8:303-308. [PubMed: 15115282]

[35]. Morishima Y, Peng HM, Lin HL, Hollenberg PF, Sunahara RK, Osawa Y, Pratt WB. Biochemistry 2005;44:16333-16340. [PubMed: 16331994]

[36]. Wang X, Medzihradszky KF, Maltby D, Correia MA. Biochemistry 2001;40:11318-11326. [PubMed: 11560479]

[37]. Scheffner M, Huibregtse JM, Vierstra RD, Howley PM. Cell 1993;75:495-505. [PubMed: 8221889]

[38]. Kaelin WG Jr. Krek W, Sellers WR, DeCaprio JA, Ajchenbaum F, Fuchs CS, Chittenden T, Li Y, Farnham PJ, Blanar MA, et al. Cell 1992;70:351-364. [PubMed: 1638635]

[39]. Wintrode PL, Makhatadze GI, Privalov PL. Proteins 1994;18:246-253. [PubMed: 8202465]

[40]. Lorick KL, Jensen JP, Fang S, Ong AM, Hatakeyama S, Weissman AM. Proc. Natl. Acad. Sci. USA 1999;96:11364-11369. [PubMed: 10500182]

[41]. Ballar P, Shen Y, Yang H, Fang S. J. Biol. Chem 2006;281:35359-35368. [PubMed: 16987818]

[42]. Morito D, Hirao K, Oda Y, Hosokawa N, Tokunaga F, Cyr DM, Tanaka K, Iwai K, Nagata AK. Mol. Biol. Cell 2008;19:1328-1336. Epub 2008 Jan 23. [PubMed: 18216283]

[43]. Jiang J, Ballinger CA, Wu Y, Dai Q, Cyr DM, Hohfeld J, Patterson C. J. Biol. Chem 2001;276:42938-42944. [PubMed: 11557750]

[44]. Qian SB, McDonough H, Boellmann F, Cyr DM, Patterson C. Nature 2006;440:551-555. [PubMed: 16554822]

[45]. Chen P, Johnson P, Sommer T, Jentsch S, Hochstrasser M. Cell 1993;74:357-369. [PubMed: 8393731]

[46]. Seufert W, Jentsch S. Embo J 1990;9:543-550. [PubMed: 2154373]

[47]. Correia MA, Sadeghi S, Mundo-Paredes E. Annu. Rev. Pharmacol. Toxicol 2005;45:439-464. [PubMed: 15822184]

[48]. Yano JK, Wester MR, Schoch GA, Griffin KJ, Stout CD, Johnson EF. J. Biol. Chem 2004;279:38091-38094. [PubMed: 15258162]

[49]. Williams PA, Cosme J, Vinkovic DM, Ward A, Angove HC, Day PJ, Vonrhein C, Tickle IJ, Jhoti H. Science 2004;305:683-686. [PubMed: 15256616]

[50]. Haas, AL. Ubiquitin. Rechsteiner, M., editor. Plenum Press; New York: 1988. p. 173-206.

Arch Biochem Biophys. Author manuscript; available in PMC 2010 June 7. 
A

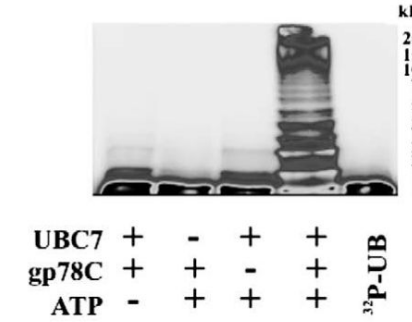

B

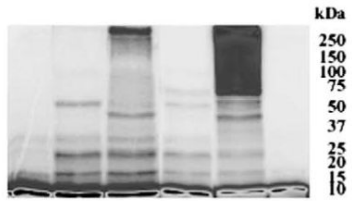

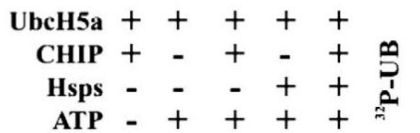

D

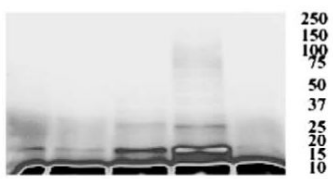

$\mathrm{UBC7}+$

ATP $-++++\underset{1}{+}$

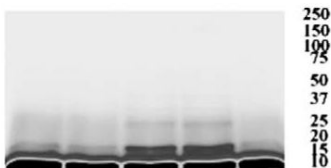

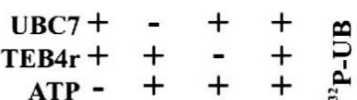

F

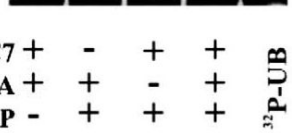

Fig. 1.

Functional competence of the reconstituted UBC7-dependent ubiquitination systems. The various combinations of $\mathrm{E} 2 \mathrm{~s}$ (UBC7 or $\mathrm{UbcH} 5 \mathrm{a}$ ) and $\mathrm{E} 3 \mathrm{~s}$ were tested for competence in forming Ub-dimers and/or polyUb chains in the presence of $\mathrm{E} 1$ and ${ }^{32} \mathrm{P}-\mathrm{Ub}$. Component dependence is shown for ATP, UBC7 or UbcH5a, and the various E3s. UbcH5a/CHIP reactions also included Hsp70, Hsc70 and Hsp40. The reactions contained E1 (0.1 $\mathrm{MM})$, UBC7 (4 $\mu \mathrm{M}),{ }^{32} \mathrm{P}-\mathrm{Ub}(20 \mu \mathrm{M})$, ATP $(4 \mathrm{mM}), \mathrm{CPK}$ (20 U/ml), CP (20 mM), $\mathrm{MgCl}_{2}$ (10 mM), DTT (1 $\mathrm{mM})$, and one of the following E3s: GST-gp78C (1 $\mu \mathrm{M})$, GST-HRD1CT1A $(10 \mu \mathrm{M})$, GSTHRD1CT1B $(10 \mu \mathrm{M})$, GST-TEB4r $(10 \mu \mathrm{M})$, or its inactive mutant GST-TEB4rC9A $(10 \mu \mathrm{M})$. UbcH5a and His-CHIP-dependent ubiquitination reactions contained: UbcH5a (1.5 $\mu \mathrm{M})$ instead of UBC7, His-CHIP $(4 \mu \mathrm{M})$ as the E3, Hsc70 $(2.5 \mu \mathrm{M})$, and Hsp40 $(2.5 \mu \mathrm{M})$, in an otherwise identical reaction mixture. The mixtures were incubated at $30{ }^{\circ} \mathrm{C}$ for $90 \mathrm{~min}$ and analyzed as detailed in Experimental Procedures. Data from a typical experiment are shown. Each experiment was conducted in its entirety at the least 3 separate times. 
A

C
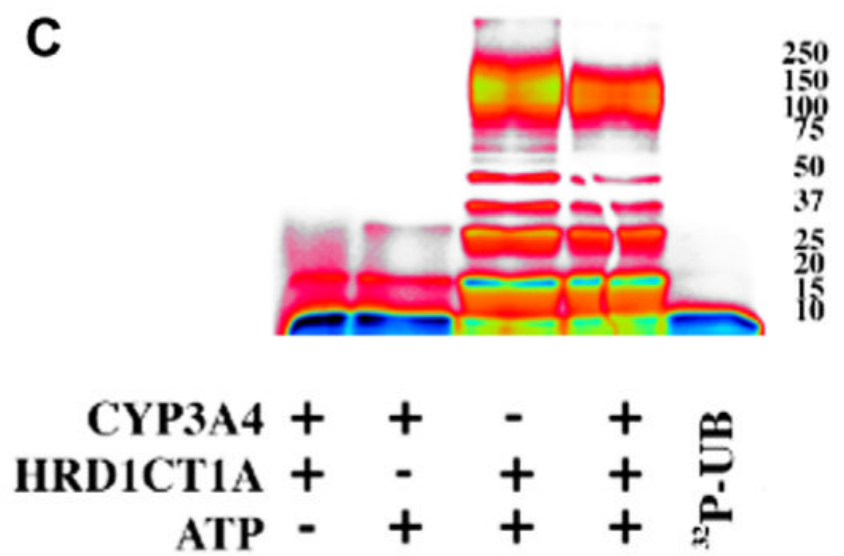

$\mathbf{E}$

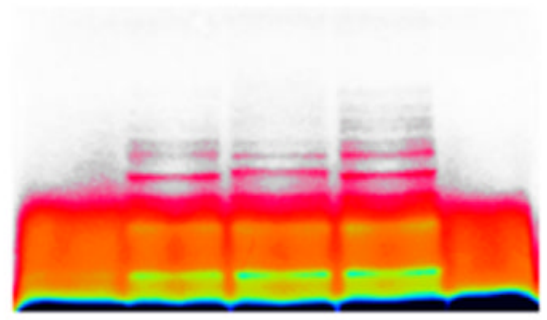

250
150
100
50
37
25
20
15

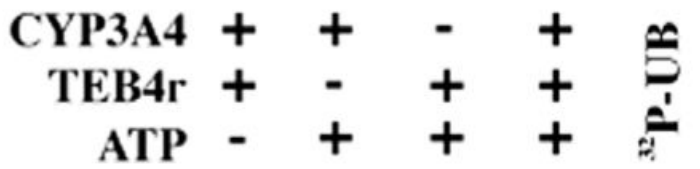

Fig. 2.
B

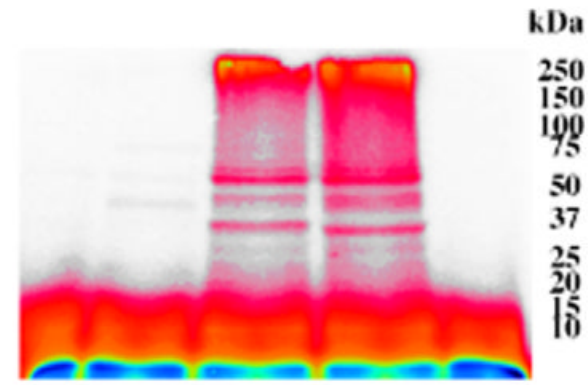

D

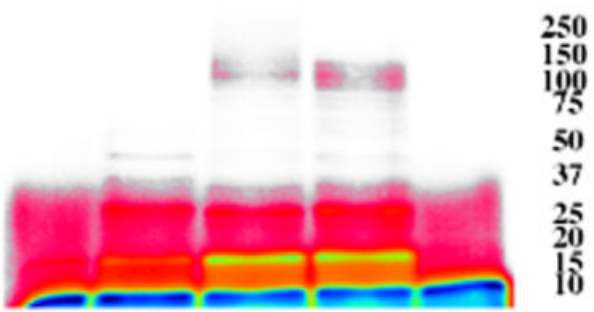

$\mathbf{F}$
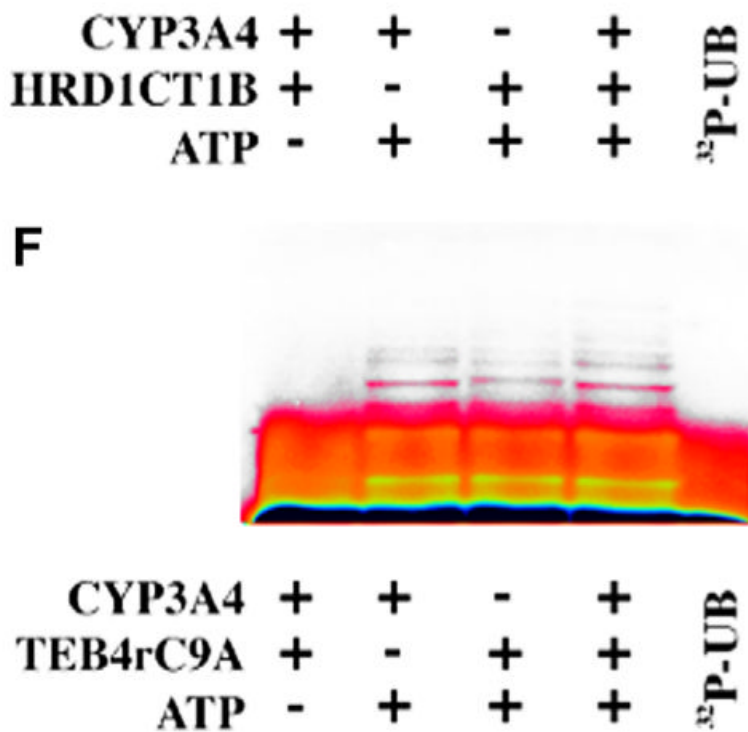

CYP3A4 reconstituted ubiquitination reactions. Each ubiquitination system identical to the complete system described in Fig. 1 was tested with and without $\mathrm{CuOOH}$-inactivated His3A4 (250 pmol) in the presence of each E3's cognate E2 at concentrations indicated in Fig. 1. E3 and ATP dependence are shown for all systems. Color contrasted images were generated using ImageQuant to enhance visualization of polyubiquitination effects. For further details see Experimental Procedures. Data from a typical experiment are shown. Each experiment was conducted in its entirety at the least 3 separate times. The color wheel intensity code for these PhosphorImager data is as follows: Black>dark blue>light 
blue $>$ green $>$ yellow $>$ orange $>$ red $>$ magenta $>$ white. (For interpretation of the references to colour in this figure legend, the reader is referred to the web version of this article.) 


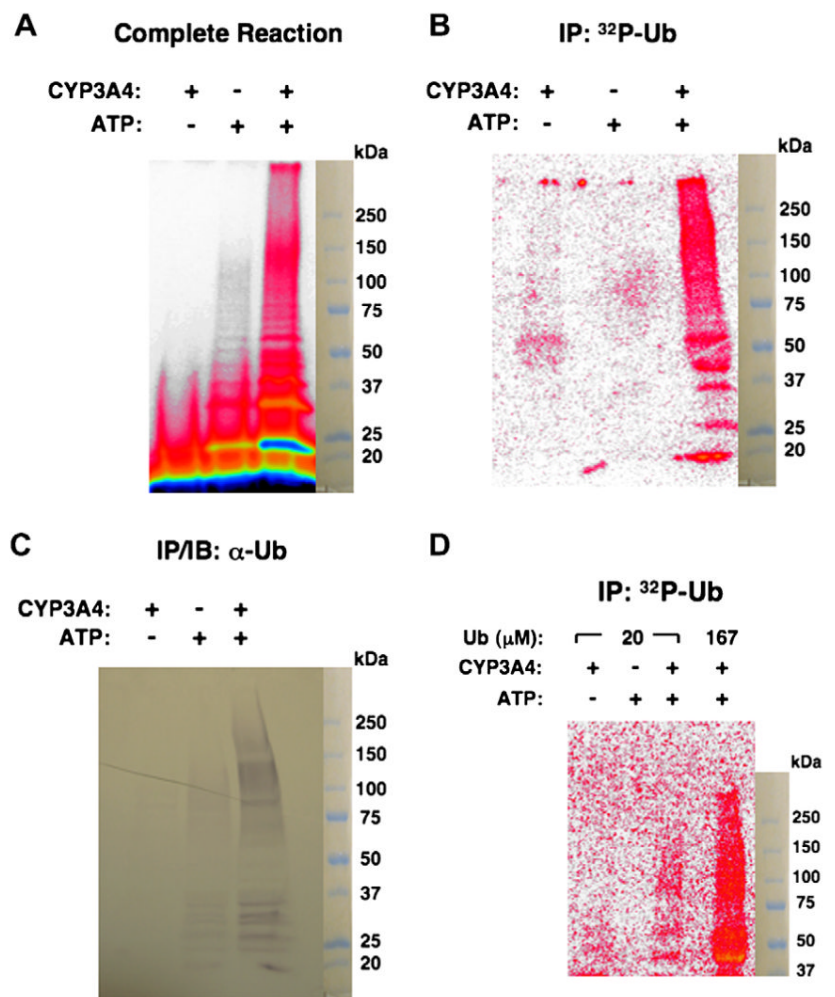

Fig. 3.

Immunoprecipitation/immunoblotting analyses of UBC7/gp78C-catalyzed CYP3A4 ubiquitination. Each UBC7/gp78C ubiquitination system identical to the complete system described in Fig. 1 except for the presence of ${ }^{32} \mathrm{P}-\mathrm{Ub}(167 \mu \mathrm{M})$, was incubated at $30{ }^{\circ} \mathrm{C}$ for 90 min with or without CYP3A4 $(250 \mathrm{pmol})$ in the presence or absence of ATP exactly as detailed (Experimental Procedures). An aliquot of each incubation was subjected to SDS-PAGE with PhosphorImager analyses (A). Other aliquots were subjected to immunoprecipitation analyses with goat anti-CYP3A4 IgGs as detailed (Experimental Procedures). Aliquots of these immunoprecipitates were then subjected to SDS-PAGE with PhosphorImager analyses (B) or immunoblotting analyses with anti-Ub IgGs as the primary antibody, followed by alkaline phosphatase as the secondary (C) as detailed [9]. A direct comparison of immunoprecipitates of aliquots of reactions carried out in the presence of Ub $20 \mu \mathrm{M}$ (Fig. 2A) or $167 \mu \mathrm{M}$ (Fig. 3A) are also shown (D). Data from a typical experiment are shown. Each experiment was conducted in its entirety at the least 3 separate times. The color wheel intensity code for these PhosphorImager data is as follows: Black $>$ dark blue $>$ light blue $>$ green $>$ yellow $>$ orange $>$ red $>$ magenta $>$ white. (For interpretation of the references to colour in this figure legend, the reader is referred to the web version of this article.) 

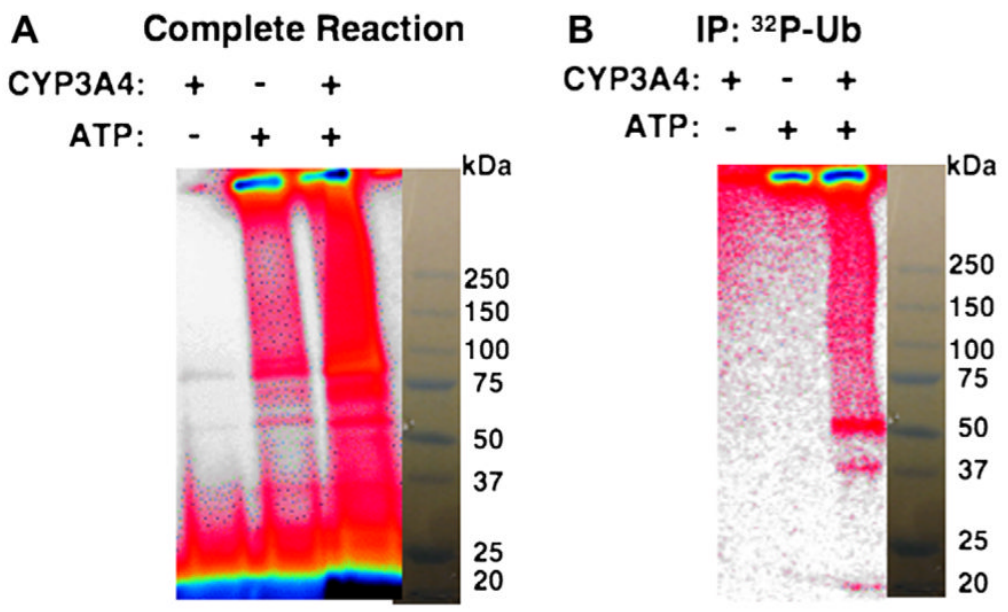

Fig. 4.

Immunoprecipitation/immunoblotting analyses of the UbcH5a/CHIP-catalyzed CYP3A4 ubiquitination. Each UbcH5a/CHIP ubiquitination system identical to the complete system described in Fig. 1 except for the presence of ${ }^{32} \mathrm{P}-\mathrm{Ub}(167 \mu \mathrm{M})$, was incubated at $30^{\circ} \mathrm{C}$ for 90 min with or without CYP3A4 $(250 \mathrm{pmol})$ in the presence or absence of ATP exactly as detailed (Experimental Procedures). An aliquot of each incubation was subjected to SDS-PAGE with PhosphorImager analyses (A). Another aliquot was subjected to immunoprecipitation analyses with goat anti-CYP3A4 IgGs as detailed (Experimental Procedures). Aliquots of these immunoprecipitates were then subjected to SDS-PAGE with PhosphorImager analyses (B). Data from a typical experiment are shown. The intense bluish green bands at the very top of either SDS-PAGE gel most likely reflect ubiquitinated CHIP and/or Hsp70, as they persist in the absence of CYP3A4 but not in the absence of ATP. Each experiment was conducted in its entirety at the least 3 separate times. The color wheel intensity code for these PhosphorImager data is as follows: Black $>$ dark blue $>$ light blue $>$ green $>$ yellow $>$ orange $>$ red $>$ magenta $>$ white. (For interpretation of the references to colour in this figure legend, the reader is referred to the web version of this article.) 


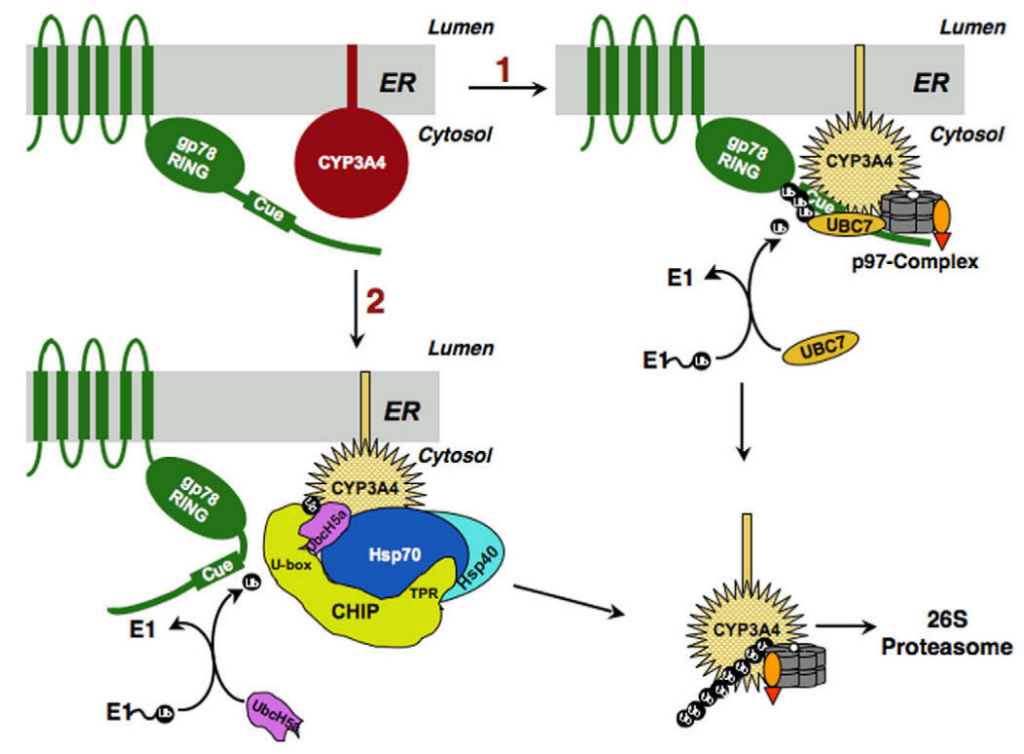

Fig. 5.

CYP3A4 ubiquitination by UBC7/gp78 and UbcH5a/CHIP systems: Plausible scenarios for physiological redundancy or functional complementarity. Following structural inactivation in vivo, ER-bound CYP3A4 could be ubiquitinated by (1) UBC7/gp78 and/or (2) UbcH5a/CHIP. The ER-polytopic gp78-RING E3 ligase catalyses polyUb chain assembly on UBC7 before transferring the elaborated polyUb chain onto accessible CYP3A4 K-residue(s). The ubiquitinated CYP3A4 is then extracted out of the ER-membrane by the AAA ATPase p97 complex and delivered to the 26S proteasome for destruction in a classical ERAD process. Alternatively or concomitantly, exposure of normally buried hydrophobic sequences within the CYP3A4 active site following structural inactivation, could target it to the cytosolic Hsp70/ Hsc70/Hsp40 molecular chaperone complex. Such Hsp70-corralled CYP3A4 becomes recognized by the cytosolic CHIP E3 ligase-tetratricopeptide repeat (TPR) motif, resulting in its subsequent ubiquitination by the UbcH5a/CHIP E3 Ub-ligase U-box domain. The ubiquitinated CYP3A4 could then be similarly delivered by the AAA ATPase p97-complex to the $26 \mathrm{~S}$ proteasome for destruction. (For interpretation of the references to colour in this figure legend, the reader is referred to the web version of this article.) 